\title{
Clinical relevance of circulating midkine in ulcerative colitis
}

\author{
Malgorzata Krzystek-Korpacka ${ }^{1, *}$, Katarzyna \\ Neubauer $^{2}$ and Malgorzata Matusiewicz ${ }^{1}$ \\ ${ }^{1}$ Department of Medical Biochemistry, Wroclaw \\ Medical University, Wroclaw, Poland \\ ${ }^{2}$ Department of Gastroenterology and Hepatology, \\ Wroclaw Medical University, Wroclaw, Poland
}

\begin{abstract}
Background: Non-invasive biochemical markers are needed to support the diagnosis of ulcerative colitis (UC), an incurable disease of unknown pathology. Midkine is an angiogenic cytokine, chemotactic towards neutrophils and macrophages, and a T-regulatory cell suppressor.

Methods: Serum midkine was measured immunoenzymatically in 93 UC patients and 108 healthy subjects, and evaluated with respect to disease status, endoscopic, inflammatory and angiogenic activity. The diagnostic value of midkine was compared to Creactive protein (CRP) using receiver operating characteristics (ROC) analysis.

Results: Midkine was higher $(p<0.0001)$ in inactive (199 $\mathrm{ng} / \mathrm{L})$ and active UC (351 $\mathrm{ng} / \mathrm{L})$ compared with controls (93 ng/L), and reflected disease activity $(r=0.427, p<0.001)$. Midkine was correlated with CRP, erythrocyte sedimentation rate (ESR), leukocytes, platelets, interleukin-6, paraoxonase-1, albumin, transferrin, iron, hemoglobin, and hematocrit. Midkine correlated with angiogenic factors: vascular endothelial growth factor-A and platelet-derived growth factorBB. As a marker of UC, midkine showed a diagnostic accuracy of $85 \%$, sensitivity of $72 \%$, specificity of $82 \%$, whereas CRP showed $83 \%, 65 \%$ and $91 \%$, respectively. As a marker of active UC, midkine showed a diagnostic accuracy of $87 \%$, sensitivity of $84 \%$, specificity of $75 \%$, whereas CRP showed $75 \%, 63 \%$ and $83 \%$, respectively. Combined assessment of midkine and CRP improved sensitivity but substantially decreased specificity.
\end{abstract}

Conclusions: UC is associated with increased circulating midkine, which corresponds with clinical, endoscopic, inflammatory and angiogenic activity, and anemia. Performance of midkine as a marker of UC or active UC was comparable to that of CRP.

Clin Chem Lab Med 2009;47:1085-90.

Keywords: C-reactive protein; disease marker; inflammation; inflammatory bowel disease; midkine; ulcerative colitis.

*Corresponding author: Malgorzata Krzystek-Korpacka, Department of Medical Biochemistry, Wroclaw Medical University, ul. Chalubinskiego 10, 50-368 Wroclaw, Poland Fax: + 487178400 85, E-mail: krzystek@bioch.am.wroc.pl Received April 27, 2009; accepted June 17, 2009

\section{Introduction}

Ulcerative colitis (UC), one of two major phenotypes of inflammatory bowel disease (IBD), is a chronic, relapsing inflammatory condition confined to the colon and rectum. The pathogenesis of IBD is not fully understood, however, it is presumed that the disease results from the coexistence of genetic predisposition, immune disregulation, epithelial barrier dysfunction, and abnormal microbial composition leading to an excessive immune response to pathogenic or functionally altered commensal enteric bacteria in susceptible subjects $(1,2)$.

The diagnosis of IBD presents some difficulties in clinical practice due to overlapping of symptoms with non-inflammatory functional bowel disorders (3). Since recognition of IBD requires extensive, invasive and expensive investigation, low-cost and noninvasive biochemical markers facilitating differential diagnosis are needed (4).

Midkine is a multifunctional, heparin-binding growth factor, transiently expressed during fetal development and subsequently during tissue repair and remodeling. However, pathological midkine overexpression has been also observed and associated with chronic inflammation, angiogenesis and neoplastic transformation $(5,6)$. Its enhanced intestinal expression has recently been reported in animal models of UC (7) and in human UC-associated adenocarcinomas (8). However, whether midkine up-regulation occurs in human UC and whether is a local phenomenon confined to inflamed gut tissue or manifests itself at systemic level remains unknown.

Thus, we assessed the concentrations of circulating midkine in patients with UC with respect to disease activity and the severity of inflammatory response. We also evaluated midkine as possible marker of UC in comparison with C-reactive protein (CRP), currently the only widely used biochemical marker.

\section{Materials and methods}

\section{Patients}

Ninety-three patients with UC presenting to the Department of Gastroenterology and Hepatology, Wroclaw Medical University, Wroclaw, Poland because of disease flare up or monitoring were enrolled in this study. Patients with coexistence of other severe systemic diseases, malignancies, liver diseases, or pregnancies were not included. Of the enrolled subjects, 55 presented with inactive and 38 with active disease. For assessment of disease activity the clinical activity index (CAI), also known as the Rachmilewitz index (RI), was used. CAI ranges from 0 to 23 and encompasses stool frequency, number of stools with blood, general wellbeing, abdominal pain/cramp, fever, extraintestinal manifestations, and laboratory tests: erythrocyte sedimentation rate 
(ESR) and hemoglobin ( $\mathrm{Hb}$ ) concentration. Endoscopic findings were evaluated as follows: a score of 0 was allocated for inactive disease, 1 for mild disease (erythema, decreased vascular pattern, mild friability), 2 for moderate disease (marked erythema, lack of vascular pattern, friability, erosions), and 3 for severe disease (spontaneous bleeding, ulceration). For reference, 108 healthy subjects were recruited: 39 healthy volunteers from the hospital staff and 69 from the Regional Center of Blood Donation and Therapy, Wroclaw, Poland. Detailed characteristics of the study population are presented in Table 1.

Anemia was recognized when $\mathrm{Hb}$ concentrations were below $120 \mathrm{~g} / \mathrm{L}$ in females and $130 \mathrm{~g} / \mathrm{L}$ in males.

\section{Analytical methods}

Blood samples were collected by venipuncture from fasting subjects into serum separator tubes and into citrate-tubes. Serum was obtained from clotted (15 min, room temperature) and centrifuged (15 $\mathrm{min}, 720 \mathrm{~g}$ ) blood. Citrated whole blood was centrifuged (15 $\mathrm{min}, 720 \mathrm{~g}$ ) and plasma was collected. Serum and plasma were stored at $-80^{\circ} \mathrm{C}$ until analysis.

Serum midkine was measured using an enzyme doubleantibody indirect immunoassays with human midkine ELISA provided by Biovendor (Brno-Modřice, Czech Republic) according to the manufacturer's instructions. An intra-assay CV for this assay is $4.5 \%$ and inter-assay CV is $6.3 \%$, analytical sensitivity is $33 \mathrm{ng} / \mathrm{L}$.

High-sensitive CRP (HS CRP) was assessed in serum by the latex particle-enhanced immunoturbidimetric method with the CRPex-HS CRP test (Good Biotech Corp., Taichung, Taiwan). Serum transferrin (TRANSF) was determined using the enhanced immunoturbidimetric method, iron with chromazurol B method, and albumin with the bromocreasol green method by Stamar (Dabrowa Gornicza, Poland). Serum interleukins- 1 and -6 (IL-1 and -6), and tumor necrosis factor (TNF)- $\alpha$ were measured using an enzyme double-antibody indirect immunoassays with PeliKine Compact human IL-1, IL-6, and TNF- $\alpha$ ELISA kits, respectively, supplied by Sanquin (Amsterdam, Netherlands).
Plasma paraoxonase-1 activity (PON1) was determined as the rate of phenylacetate (ChemService Inc., West Chester, PA, USA) hydrolysis followed spectrophotometrically as the increase in absorbance at $270 \mathrm{~nm}$ at $25^{\circ} \mathrm{C}$.

The serum concentration of vascular endothelial growth factor (VEGF)-A was assayed by an immunoenzymatic method with tests provided by IBL (Hamburg, Germany). Serum platelet-derived growth factor (PDGF)-BB was measured with Human PDGF-BB Stratikine ELISA provided by Strathmann Biotec GmbH (Hamburg, Germany).

Other laboratory indices were assessed with routine automated procedures.

\section{Statistical analysis}

The distribution of data was analyzed with the D'AgostinoPearson test. Non-normally distributed data (midkine) are presented as medians and compared with Mann-Whitney U-test or Kruskal-Wallis test (multigroup comparisons). Normally distributed data (either raw or log-transformed) are presented as means and analyzed with t-test for independent samples or one-way ANOVA. Means and medians are presented with $95 \%$ confidence intervals $(\mathrm{Cl})$. Correlation analysis was conducted using Spearman's rank correlation test. Second order correlation coefficients were calculated in order to obtain the net correlation for four interrelated variables. Frequency analysis was conducted with the $\chi^{2}$ test. All calculated $p$-values were two-sided and $p \leq 0.05$ was considered statistically significant.

The diagnostic value of midkine and CRP was evaluated using receiver operating characteristics (ROC) curve analysis. The overall performance was expressed as the area under the ROC curve (AUC) with $95 \% \mathrm{Cl}$ and p-statistics for the difference between calculated $A U C$ and $A U C=0.5$ (marker without discriminative power). A cut-off value corresponding to the highest accuracy was determined and the related sensitivities and specificities as well as likelihood ratios for positive and negative results were calculated. In the evaluation of combined midkine and CRP assessment, the rule "at least 1 of the 2 markers positive" was applied as the criterion for test positivity.

Table 1 Characteristics of the study population.

\begin{tabular}{|c|c|c|c|c|}
\hline Index & Controls & Inactive UC & Active UC & $\mathrm{p}$-Value \\
\hline Age, years (range) & $40.8(21-66)$ & $47.7(18-78)$ & $43.3(18-79)$ & $0.198^{t}$ \\
\hline Gender, M/F & $44 / 64$ & $32 / 23$ & $20 / 18$ & $0.087 x^{2}$ \\
\hline UC duration, years (range) & - & $7.0(5.0-10.0)$ & $3.5(1.5-11.3)$ & $0.303^{\mathrm{M}}$ \\
\hline Age at onset, years (range) & - & $40.6(35.7-45.6)$ & $33.4(28.2-38.6)$ & $0.048^{t}$ \\
\hline Albumin, g/L & $48.3(47.2-49.5)$ & $45.9(44.7-48.2)^{a}$ & $43.9(39.4-45.5)^{a, b}$ & $<0.0001^{\mathrm{K}}$ \\
\hline $\mathrm{CRP}, \mathrm{mg} / \mathrm{L}$ & $0(0-1.04)$ & $12.87(5.05-28.10)^{a}$ & $78.31(20.95-139.35)^{a, b}$ & $<0.0001^{\mathrm{K}}$ \\
\hline $\mathrm{ESR}, \mathrm{mm} / \mathrm{h}$ & $8.7(4.6-16.2)^{\mathrm{c}}$ & $12.3(9.5-15.8)$ & $28.7(21.9-37.7)^{a, b}$ & $<0.001^{\mathrm{A}}$ \\
\hline $\mathrm{Hb}, \mathrm{g} / \mathrm{L}$ & $138(128-147)^{\mathrm{c}}$ & $135(127-138)$ & $117(104-126)^{a, b}$ & $0.0001^{\mathrm{K}}$ \\
\hline HCT, \% & $41.6(38.7-44.5)^{\mathrm{c}}$ & $41.0(39.1-42.0)$ & $35.5(33.1-38.5)^{a, b}$ & $0.0001^{\mathrm{K}}$ \\
\hline $\mathrm{RBC}, \times 10^{9} / \mathrm{L}$ & $4.72(4.31-5.12)^{\mathrm{c}}$ & $4.59(4.45-4.73)$ & $4.18(3.72-4.51)^{a, b}$ & $0.002^{\mathrm{K}}$ \\
\hline Iron, $\mu \mathrm{mol} / \mathrm{L}$ & $18.2(16.1-20.4)$ & $16.6(14.4-18.8)$ & $10.9(8.8-12.9)^{a, b}$ & $<0.001^{\mathrm{A}}$ \\
\hline $\mathrm{IL}-1, \mathrm{ng} / \mathrm{L}$ & $0.79(0-2.42)$ & $0.88(0.59-1.67)$ & $0.88(0.34-3.14)$ & $0.538^{\mathrm{K}}$ \\
\hline IL-6, ng/L & $0.68(0.54-0.76)$ & $1.48(0.93-2.52)^{a}$ & $4.09(1.82-6.18)^{a, b}$ & $<0.0001^{\mathrm{K}}$ \\
\hline TNF- $\alpha, n g / L$ & $0.62(0.21-1.59)$ & $0.44(0.19-1.40)$ & $0.53(0.21-1.19)$ & $0.979^{\mathrm{K}}$ \\
\hline $\mathrm{PLT}, \times 10^{9} / \mathrm{L}$ & $241(197-295)^{\mathrm{c}}$ & $274(253-292)$ & $349(304-416)^{a, b}$ & $<0.001^{\mathrm{K}}$ \\
\hline TRANSF, g/L & $2.85(2.67-3.03)$ & $2.86(2.60-3.03)$ & $2.45(2.16-2.60)^{a, b}$ & $<0.001^{\mathrm{K}}$ \\
\hline WBC,$\times 10^{9} / \mathrm{L}$ & $5.25(4.44-6.20)^{\mathrm{c}}$ & $6.20(5.71-7.20)$ & $8.40(6.64-9.91)^{a, b}$ & $0.008^{\mathrm{K}}$ \\
\hline
\end{tabular}

Unless otherwise stated, data are presented with $95 \% \mathrm{Cl}$ around mean/median. a Significantly different from controls; ${ }^{\mathrm{b}}$ significantly different from inactive UC; ${ }^{c}$ not evaluated in blood donors; ${ }^{2}$ Chi-square test; ${ }^{\mathrm{K}}$ Kruskal-Wallis test; ${ }^{\mathrm{A}} \mathrm{ANOVA}$; ${ }_{\mathrm{t}} \mathrm{i}$-test for independent samples; ${ }^{M} M a n n-W h i t n e y ~ U-t e s t . ~ U C$, ulcerative colitis; M/F, males/females ratio; ESR, erythrocyte sedimentation rate; Hb, hemoglobin; HCT, hematocrit; RBC, red blood cell count; IL, interleukin; TNF, tumor necrosis factor; PLT, platelet count; TRANSF, transferrin; WBC, white blood cell count; CRP, C-reactive protein. 
The entire statistical analysis was conducted with MedCalc $^{\circledR}$ Version 10.1.0.0 software (Mariakerke, Belgium) with the exception of partial correlation analysis for which VassarStats was applied (available from http://faculty.vassar. edu/lowry/VassarStats.html).

\section{Ethical considerations}

The study protocol was approved by the medical Ethics Committee of our University and was in accordance with the ethical standards formulated in the Helsinki Declaration of 1975. Informed consent was obtained from all subjects.

\section{Results}

\section{Circulating midkine in UC}

Midkine concentrations in patients with both active and quiescent UC were significantly increased compared to healthy subjects (Figure 1). Midkine correlated directly with disease clinical activity and, although weakly, with endoscopy findings (Table 2).

Midkine also correlated directly with CRP, ESR, white blood cell count (WBC), and platelet count $(\mathrm{PLT})$. It correlated indirectly with negative acute-

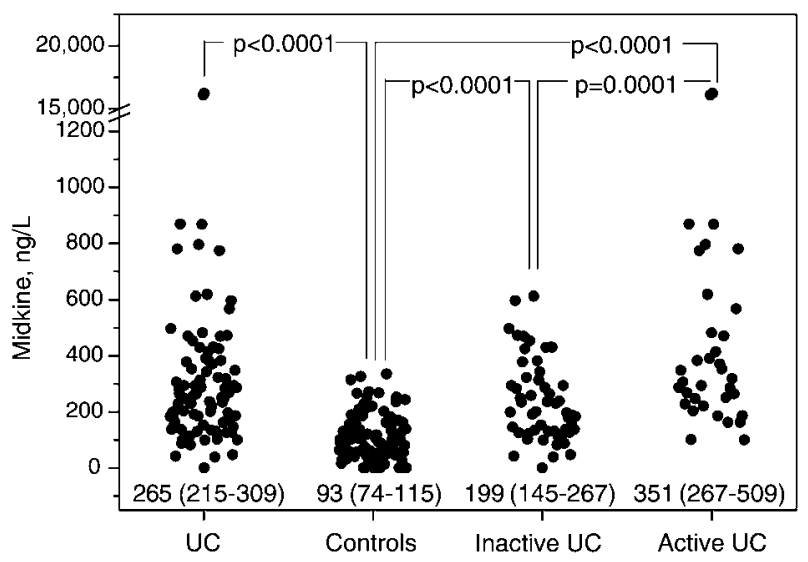

Figure 1 Serum midkine concentrations in patients with inactive and active ulcerative colitis (UC) compared to healthy subjects.

Median midkine concentrations are shown with $95 \%$ confidence intervals. phase response proteins - albumin and TRANSF. Of the proinflammatory cytokines, midkine, similarly to CRP and ESR, correlated with IL-6, but not with TNF$\alpha$ and IL-1. A negative correlation with the activity of PON1 was also observed (Tables 2 and 3).

Since midkine, disease activity, ESR and CRP were all interrelated, we calculated the second order partial correlation coefficients to obtain the netto correlation between factors. These were as follows: for midkine and disease activity $r=0.272, p=0.009$, for midkine and CRP $r=0.115, p=0.278$, for midkine and ESR $r=0.173, p=0.101$. Partial correlation coefficients for midkine and endoscopy findings, following the removal of the effects of CRP and ESR, were $r=0.054$, $\mathrm{p}=0.611$.

Midkine concentrations were significantly higher in anemic patients with UC compared with those without anemia [377 ng/L (95\% Cl: 288-565) vs. 202 ng/L (169-260), $p=0.0001]$. Midkine was negatively correlated with $\mathrm{Hb}$ and iron concentrations and with hematocrit (HCT) (Table 3). Patients who reported substantial involuntary weight loss had insignificantly higher midkine concentrations compared with those who did not [294 ng/L (189-798) vs. $256 \mathrm{ng} / \mathrm{L}$ (183-431), $p=0.397$ ].

Midkine concentrations correlated directly with concentrations of angiogenic factors - VEGF-A and PDGF-BB (Table 3).

No significant correlation was found between midkine concentrations and patient age $(r=-0.128$, $p=0.222)$, age at onset of disease $(r=-0.073, p=$ $0.520)$ or disease duration $(r=-0.207, p=0.060)$. There was no significant differences $(p=0.401)$ in cytokine concentrations between female [294 ng/L (219-382)] and male [242 ng/L (185-297)] participants.

\section{Midkine as a possible disease marker in UC}

Midkine as a marker of the presence of UC (both active and inactive) had an $A \cup C=0.848$ (0.791-0.895), $\mathrm{p}=0.0001$. Using $178 \mathrm{ng} / \mathrm{L}$ as a cut-off value, the marker showed a sensitivity of $72 \%$ and specificity of $82 \%$. The likelihood ratio for a positive result was 3.89, and the likelihood ratio for a negative result was 0.34 . CRP had an $A \cup C=0.830(0.760-0.886), p=0.0001$. Using $10.4 \mathrm{mg} / \mathrm{L}$ as a cut-off value, CRP had a sensitivity of

Table 2 Relationship between disease activity, inflammatory response and circulating midkine in patients with ulcerative colitis compared with CRP and ESR.

\begin{tabular}{llll}
\hline Variables & Midkine & CRP & ESR \\
\hline CAI & $r=0.427, p<0.001$ & $r=0.360, p=0.007$ & $r=0.497, p=0.0001$ \\
Endoscopy & $r=0.266, p=0.016$ & $r=0.422, p<0.001$ & $r=0.498, p<0.0001$ \\
CRP & $r=0.326, p=0.004$ & - & $r=0.527, p<0.0001$ \\
ESR & $r=0.401, p<0.001$ & - & - \\
WBC & $r=0.365, p<0.001$ & $r=0.399, p<0.001$ & $r=0.406, p=0.0001$ \\
PLT & $r=0.394, p<0.001$ & $r=0.286, p=0.012$ & $r=0.564, p<0.0001$ \\
IL-1 & $r=0.024, p=0.849$ & $r=0.115, p=0.397$ & $r=-0.010, p=0.935$ \\
IL-6 & $r=0.350, p=0.006$ & $r=0.527, p=0.0001$ & $r=0.578, p<0.0001$ \\
TNF- $\alpha$ & $r=-0.046, p=0.713$ & $r=-0.413, p=0.002$ & $r=0.004, p=0.976$ \\
PON1 & $r=-0.473, p=0.0001$ & $r=-0.362, p=0.004$ \\
\hline
\end{tabular}

CRP, C-reactive protein; ESR, erythrocyte sedimentation rate; CAl, clinical activity index (Rachmilewitz Index); WBC, white blood cell count; PLT, platelet count; IL, interleukin; TNF, tumor necrosis factor; PON1, paraoxonase 1. 
Table 3 Relationship between nutritional indices, hematological parameters, angiogenic factors and circulating midkine in patients with ulcerative colitis compared with CRP and ESR.

\begin{tabular}{llll}
\hline Variables & Midkine & CRP & ESR \\
\hline Albumin & $r=-0.368, p<0.001$ & $r=-0.411, p<0.001$ & $r=-0.362, p<0.001$ \\
TRANSF & $r=-0.295, p=0.007$ & $r=-0.296, p=0.013$ & $r=-0.288, p=0.010$ \\
Iron & $r=-0.312, p=0.005$ & $r=-0.601, p<0.0001$ & $r=-0.409, p<0.001$ \\
Hb & $r=-0.363, p<0.001$ & $r=-0.365, p=0.001$ & $r=-0.470, p<0.0001$ \\
HCT & $r=-0.357, p<0.001$ & $r=-0.353, p=0.002$ & $r=-0.483, p<0.0001$ \\
RBC & $r=-0.175, p=0.099$ & $r=-0.180, p=0.114$ & $r=-0.257, p=0.016$ \\
MCV & $r=-0.275, p=0.009$ & $r=-0.262, p=0.022$ & $r=-0.328, p=0.002$ \\
PDGF-BB & $r=0.359, p=0.006$ & $r=0.127, p=0.374$ & $r=0.277, p=0.036$ \\
VEGF-A & $r=0.402, p=0.008$ & $r=-0.053, p=0.837$ & $r=0.190, p=0.462$ \\
\hline
\end{tabular}

CRP, C-reactive protein; ESR, erythrocyte sedimentation rate; TRANSF, transferrin; Hb, hemoglobin; HCT, hematocrit; RBC, red blood cell count; MCV, mean corpuscular volume; PDGF, platelet-derived growth factor; VEGF, vascular endothelial growth factor.

$65 \%$ and specificity of $91 \%$. The likelihood ratio for a positive result was 6.92 , and 0.38 for a negative result. Combining both markers increased test sensitivity to $91 \%$ but decreased specificity to $70 \%$. Corresponding likelihood ratios were 3.0 and 0.13 for positive and negative test results, respectively.

Midkine as a marker for patients with active UC had an $A \cup C=0.870(0.816-0.913), p=0.0001$. Using a cutoff value of $202 \mathrm{ng} / \mathrm{L}$, midkine had a sensitivity of $84 \%$ and specificity of $75 \%$. The likelihood ratio for a positive result was 3.35 , and 0.21 for a negative result. CRP had an $A U C=0.751$ (0.674-0.817), $p=0.0001$. Using a cut-off value of $10.4 \mathrm{mg} / \mathrm{L}, \mathrm{CRP}$ had a sensitivity of $63 \%$ and specificity of $83 \%$. The likelihood ratio for a positive result was 3.60 and 0.45 for a negative result. Combining both markers increased test sensitivity to $87 \%$ but decreased specificity to $47 \%$. Corresponding likelihood ratios were 1.65 and 0.28 for positive and negative test results, respectively.

\section{Discussion}

The up-regulation of midkine expression has been observed frequently in a number of pathological conditions (9). At the systemic level, however, increases in midkine have been reported almost exclusively in cancer $(10,11)$. To date, rheumatoid arthritis represents the only inflammatory disease in which an increase in circulating midkine concentrations has been observed (12). Thus, our paper supports the link between midkine and inflammation at the systemic level.

Tokuyama et al. (8) demonstrated increased midkine expression in colon adenocarcinoma, which increased along with tumor progression. Midkine has been overexpressed in sporadic and to a lesser degree, UC-associated tumors. These authors reported increased concentrations of midkine in UC-regenerative mucosa. This finding implies midkine involvement not only in UC-associated neoplastic transformation, but also in the healing process of UC. The latter finding corroborates the observations of Yuki et al. (7), who demonstrated midkine overexpression in a rat model of disease and suggested its involvement in reepithelialization and modulation of the cell-matrix interactions during the process of gut repair. Midkine contributions to the healing process in UC is further supported by its participation in the healing of gastric deep ulcers (13).

Our study shows up-regulation of midkine in UC at the systemic level. Midkine expression has been found in epithelial cells of intestinal mucosa and endothelial cells of gut blood vessels (8). Also, immune cells infiltrating inflamed tissue (14), such as lymphocytes (15), neutrophiles (16), and macrophages (17), may contribute to increased concentration of midkine. Platelets, which can selectively scavenge angiogenic factors and release them upon activation (18), may be another possible source of this cytokine. Accordingly, we observed increased PLTs in active UC, as well as direct correlation between midkine and platelet or leukocyte counts.

We demonstrated that midkine was slightly increased in patients in remission, which is in agreement with its suggested role in gut healing. However, midkine elevations were associated primarily with flare up of disease and correlated directly with disease activity indices, which may imply its involvement in the pathogenesis of UC. The association with disease activity was valid for its clinical rather than endoscopic evaluation, as revealed by the removal of the effects exerted by CRP and ESR. Concerning the inclusion of $\mathrm{Hb}$ in the $\mathrm{CAI}$, the association of midkine with hematological variables, consistent with our previous studies $(19,20)$, may account for the relationship of midkine with clinical activity of UC.

Midkine moderately reflected the systemic inflammatory response, being correlated with immune cell counts, acute-phase proteins and cytokines/growth factors with proinflammatory properties. However, except for PON1, the observed associations were weaker than those of CRP and ESR, the inflammatory indices of clinical relevance in UC (4). Similar to Crohn's disease (CD) (20) and gastrointestinal tract cancers (21), midkine increases in UC correlated with a drop in the activity of PON1. This enzyme plays a role in the gastrointestinal tract as a local detoxifier, antioxidant, immunomodulator, and/or quorumquenching factor (22). Our results are in agreement with postulated involvement of midkine in the perpetuation and exacerbation of the inflammatory 
response $(5,6)$. It may play a role in recruitment of neutrophils and macrophages into inflamed tissue $(23,24)$, and up-regulation of synthesis of inflammatory mediators (25-27). Animal studies have revealed that several inflammation-related phenomena were substantially alleviated in midkine-deficient animals $(12,25,28-32)$. The mechanisms mentioned above may also operate in the involvement of midkine in the pathogenesis of UC since the accumulation of neutrophiles and monocytes associated with aberrant inflammatory response is essential for development of UC (14). Recently, midkine was found to act as a suppressor of $T$ regulatory lymphocytes (Treg) (31), the dysfunction of which contributes to immune abnormalities of mucosa $(33,34)$. Wang et al. (31) demonstrated that attenuated symptoms of encephalomyelitis in midkine-deficient animals resulted from increased Treg cell population. Midkine was found to decrease cell number in a dose-dependent manner, whereas the administration of anti-midkine RNA aptamers expanded the Treg cell population.

Furthermore, although immune-driven angiogenesis seems to be a secondary event to abnormal immune response/barrier dysfunction in the pathogenesis of IBD, it substantially contributes to the chronic character of the disease (34). Several of the properties of midkine support angiogenesis - it exhibits mitogenic, differentiation, and chemotactic activity towards vascular endothelial and smooth muscle cells $(26,29,35)$ and facilitates remodeling of extracellular matrix (36). Also, the recruitment of inflammatory cells secreting angiogenic factors indirectly supports angiogenesis (37). Accordingly, in the present study midkine increases corresponded with increasing concentrations of VEGF-A and PDGF-BB, the prominent angiogenic factors involved in the pathogenesis of IBD (38).

The need for biochemical markers facilitating clinical decision-making has recently been stressed (4). Especially non-invasive and economical markers for supporting the differential diagnosis of UC from subjects with non-inflammatory functional bowel disorders are needed. Therefore, apart from ability of midkine to detect UC, we evaluated the ability to distinguish patients with active UC from healthy subjects and patients with quiescent disease, the latter group being a surrogate for subjects with non-inflammatory bowel disorders. The overall performance of midkine as a marker for UC was similar to that of CRP. Midkine proved to be a more sensitive, while CRP proved to be a more specific indicator of UC. However, the likelihood ratio for a positive result obtained with CRP was twice that of midkine, demonstrating that CRP is superior in ruling in disease. More substantial differences in midkine and CRP performance were observed in distinguishing active disease - midkine accuracy was considerably greater than that of CRP. Again, midkine proved to be a more sensitive, while CRP proved to be a more specific marker. Simultaneous measurement of both factors resulted in insignificant improvement in sensitivity and considerable worsening of specificity. The fact that midkine per- formance as a marker for UC was not substantially better than that for CRP implies its limited usefulness for clinical application in case of this disease. Our results obtained for midkine as a disease marker in CD (20), superior to those obtained for UC, imply that midkine better reflects inflammation in CD than in UC. Correspondingly, CD has been found to be associated with a greater CRP response than in UC (39). This may reflect different pathophysiology involved in the development of both diseases.

Taking into account the findings of Tokuyama et al. (8) on enhanced expression of midkine in UC-associated dysplasia, it cannot be excluded that midkine increases in some patients with clinically and endoscopically inactive disease may reflect UC-associated neoplastic transformation. Presently, there is no other instrument for oncologic surveillance of UC patients other than invasive and expensive endoscopy associated with histopathological examination of biopsy specimen. In this respect, the possible use of midkine as an early cancer marker for surveillance of UC patients is intriguing and warrants further investigations.

\section{Conflict of interest statement}

Hereby, the authors certify that there is no conflict of interest of any kind, which would concern the studies described in the submitted manuscript. We did not accept any funding or support from an organization that may in any way gain or lose financially from the results of our study. None of the authors has been employed by an organization that may in any way gain or lose financially from the results of our study. We have no other conflicting interests.

\section{Acknowledgements}

The authors would like to thank Dr. Elzbieta Klausa from the Regional Center of Blood Donation and Therapy, Wroclaw, Poland for the supply of control sera.

\section{References}

1. Kucharzik T, Maaser $C$, Lügering $A$, Kagnoff $M$, Mayer $L$, Targan $S$, et al. Recent understanding of IBD pathogenesis: implications for future therapies. Inflamm Bowel Dis 2006;12:1068-83.

2. Packey $C D$, Sartor RB. Interplay of commensal and pathogenic bacteria, genetic mutations and immunoregulatory defects in the pathogenesis of inflammatory bowel diseases. J Intern Med 2008;263:597-606.

3. Tibble JA, Bjarnason I. Non-invasive investigation of inflammatory bowel disease. World J Gastroenterol 2001; $7: 460-5$.

4. Vermeire S, Van Assche G, Rutgeerts P. Laboratory markers in IBD: useful, magic, or unnecessary toys? Gut 2006; 55:426-31.

5. Muramatsu T. Midkine and pleiotrophin: two related proteins involved in development, survival, inflammation and tumorigenesis. J Biochem 2002;132:359-71.

6. Kadomatsu K, Muramatsu T. Midkine and pleiotrophin in neural development and cancer. Cancer Lett 2004;204: 127-43. 
7. Yuki T, Ishihara S, Rumi MA, Ortega-Cava CF, Kadowaki $\mathrm{Y}$, Kazumori $\mathrm{H}$, et al. Increased expression of midkine in the rat colon during healing of experimental colitis. Am J Physiol Gastrointest Liver Physiol 2006;291:G735-43.

8. Tokuyama W, Mikami T, Fujiwara M, Matsui T, Okayasu I. Midkine expression in colorectal tumors: correlation with Ki-67 labeling in sporadic, but not ulcerative colitisassociated ones. Pathol Int 2007;57:260-7.

9. Kadomatsu K. The midkine family in cancer, inflammation and neural development. Nagoya J Med Sci 2005; 67:71-82.

10. Ikematsu S, Yano A, Aridome K, Kikuchi M, Kumai H, Nagano $\mathrm{H}$, et al. Serum midkine levels are increased in patients with various types of carcinomas. $\mathrm{Br} \mathrm{J}$ Cancer 2000;83:701-6.

11. Krzystek-Korpacka M, Matusiewicz M, Diakowska D, Grabowski K, Blachut K, Kustrzeba-Wojcicka I, et al. Serum midkine depends on lymph node involvement and correlates with circulating VEGF-C in oesophageal squamous cell carcinoma. Biomarkers 2007;12:403-13.

12. Maruyama K, Muramatsu $H$, Ishiguro N, Muramatsu T. Midkine, a heparin binding growth factor, is fundamentally involved in the pathogenesis of rheumatoid arthritis. Arthritis Rheum 2004;50:1420-9.

13. Maekawa T, Waki S, Okada A, Fukui H, Kinoshita $Y$, Chiba T. Midkine gene expression in the healing process of gastric ulcer. J Lab Clin Med 1999;133:349-52.

14. Kruidenier L, Verspaget HW. Review article: oxidative stress as a pathogenic factor in inflammatory bowel disease - radicals or radiculous? Aliment Pharmacol Ther 2002;16:1997-2015.

15. Callebaut C, Nisole S, Briand JP, Krust B, Hovanessian AG. Inhibition of HIV infection by the cytokine midkine. Virology 2001;281:248-64.

16. Schruefer R, Sulyok S, Schymeinsky J, Peters T, Scharffetter-Kochanek K, Walzog B. The proangiogenic capacity of polymorphonuclear neutrophils delineated by microarray technique and by measurement of neovascularization in wounded skin of CD18-deficient mice. J Vasc Res 2006;43:1-11.

17. Narita $\mathrm{H}$, Chen $\mathrm{S}$, Komori K, Kadomatsu K. Midkine is expressed by infiltrating macrophages in in-stent restenosis in hypercholesterolemic rabbits. J Vasc Surg 2008; 47:1322-9.

18. Klement GL, Yip T-T, Cassiola F, Kikuchi L, Cervi D, Podust $\mathrm{V}$, et al. Platelets actively sequester angiogenesis regulators. Blood 2008; doi:10.1182/blood-2008-06159541.

19. Krzystek-Korpacka M, Matusiewicz M, Diakowska D, Grabowski K, Blachut K, Kustrzeba-Wojcicka I, et al. Even a mild anemia is related to tumor aggressiveness mediated by angiogenic factors. Exp Oncol 2009;31:52-6.

20. Krzystek-Korpacka M, Neubauer K, Matusiewicz M. Circulating midkine in Crohn's disease: clinical implications. Inflamm Bowel Dis 2009.doi:10.1002/ibd.21011.

21. Krzystek-Korpacka M, Boehm D, Matusiewicz M, Diakowska D, Grabowski K, Gamian A. Paraoxonase 1 (PON1) status in gastroesophageal malignancies and associated paraneoplastic syndromes - connection with inflammation. Clin Biochem 2008;41:804-11.

22. Rothem L, Hartman C, Dahan A, Lachter J, Eliakim R, Shamir R. Paraoxonases are associated with intestinal inflammatory diseases and intracellularly localized to the endoplasmic reticulum. Free Radic Biol Med 2007;43: 730-9.

23. Hayashi K, Kadomatsu K, Muramatsu T. Requirement of chondroitin sulfate/dermatan sulfate recognition in midkine-dependent migration of macrophages. Glycoconj J 2001;18:401-6.

24. Takada T, Toriyama K, Muramatsu H, Song XJ, Torii S, Muramatsu T. Midkine, a retinoic acid-inducible heparinbinding cytokine in inflammatory responses: chemotactic activity to neutrophils and association with inflammatory synovitis. J Biochem 1997;122:453-8.

25. Sato W, Kadomatsu K, Yuzawa Y, Muramatsu H, Hotta $\mathrm{N}$, Matsuo S, et al. Midkine is involved in neutrophil infiltration into the tubulointerstitium in ischemic renal injury. J Immunol 2001;167:3463-9.

26. Sumi Y, Muramatsu H, Takei $Y$, Hata K, Ueda M, Muramatsu T. Midkine, a heparin-binding growth factor promotes growth and glycosaminoglycan synthesis of endothelial cells through its action on smooth muscle cells in an artificial blood vessel model. J Cell Sci 2002; 115:2659-66.

27. Yamada H, Inazumi T, Tajima S, Muramatsu H, Muramatsu T. Stimulation of collagen expression and glycosaminoglycan synthesis by midkine in human skin fibroblasts. Arch Dermatol Res 1997;289:429-33.

28. Kawai H, Sato W, Yuzawa Y, Kosugi T, Matsuo S, Takei $Y$, et al. Lack of the growth factor midkine enhances survival against cisplatin-induced renal damage. Am J Pathol 2004;165:1603-12.

29. Horiba M, Kadomatsu K, Nakamura E, Muramatsu H, Ikematsu S, Sakuma S, et al. Neointima formation in a restenosis model is suppressed in midkine-deficient mice. $\mathrm{J}$ Clin Invest 2000;105:489-95.

30. Narita H, Chen S, Komori K, Kadomatsu K. Midkine is expressed by infiltrating macrophages in in-stent restenosis in hypercholesterolemic rabbits. J Vasc Surg 2008; 47:1322-9.

31. Wang J, Takeuchi H, Sonobe $Y$, Jin S, Mizuno T, Miyakawa $S$, et al. Inhibition of midkine alleviates experimental autoimmune encephalomyelitis through the expansion of regulatory $T$ cell population. Proc Natl Acad Sci USA 2008;105:3915-20.

32. Kosugi T, Yuzawa Y, Sato W, Arata-Kawai H, Suzuki N, Kato $\mathrm{N}$, et al. Midkine is involved in tubulointerstitial inflammation associated with diabetic nephropathy. Lab Invest 2007:87:903-13.

33. Coombes JL, Robinson NJ, Maloy KJ, Uhlig HH, Powrie F. Regulatory T cells and intestinal homeostasis. Immunol Rev 2005;204:184-94

34. Scaldaferri F, Fiocchi C. Inflammatory bowel disease: progress and current concepts of etiopathogenesis. $J$ Dig Dis 2007;8:171-8.

35. Reynolds PR, Mucenski ML, Le Cras TD, Nichols WC, Whitsett JA. Midkine is regulated by hypoxia and causes pulmonary vascular remodeling. J Biol Chem 2004;279: 37124-32.

36. Kojima S, Inui T, Kimura T, Sakakibara S, Muramatsu $H$, Amanuma $\mathrm{H}$, et al. Synthetic peptides derived from midkine enhance plasminogen activator activity in bovine aortic endothelial cells. Biochem Biophys Res Commun 1995;206:468-73.

37. Yu JL, Rak JW. Host microenvironment in breast cancer development: inflammatory and immune cells in tumor angiogenesis and arteriogenesis. Breast Cancer Res 2003;5:83-8.

38. Chidlow JH Jr, Shukla D, Grisham MB, Kevil CG. Pathogenic angiogenesis in IBD and experimental colitis: new ideas and therapeutic avenues. Am J Physiol Gastrointest Liver Physiol 2007;293:G5-18.

39. Vermeire S, Van Assche G, Rutgeerts P. C-reactive protein as a marker for inflammatory bowel disease. Inflamm Bowel Dis 2004;10:661-5. 